\title{
Fast Computation of Polyharmonic B-Spline Autocorrelation Filters
}

\author{
Yann Barbotin, Student Member, IEEE, Dimitri Van De Ville, Member, IEEE, Thierry Blu, Senior Member, IEEE, \\ and Michael Unser, Fellow, IEEE
}

\begin{abstract}
A fast computational method is given for the Fourier transform of the polyharmonic B-spline autocorrelation sequence in $d$ dimensions. The approximation error is exponentially decaying with the number of terms taken into account. The algorithm improves speed upon a simple truncated-sum approach. Moreover, it is virtually independent of the spline's order. The autocorrelation filter directly serves for various tasks related to polyharmonic splines, such as interpolation, orthonormalization, and wavelet basis design.
\end{abstract}

Index Terms-Autocorrelation sequence, Epstein zeta function, polyharmonic B-splines.

\section{INTRODUCTION}

$\mathbf{T}$ HE Laplacian has a very special status in image processing because it is one of the few operators that are invariant with respect to the three fundamental coordinate transformations: translation, dilation, and rotation. In fact, the complete family of such real-valued operators reduces to the fractional iterates of the Laplacian $(-\Delta)^{\gamma / 2}[1]$, whose distributional Fourier-domain definition is

$$
\forall f \in \mathcal{S}^{\prime}, \quad(-\Delta)^{\frac{\gamma}{2}} f(\boldsymbol{x}) \stackrel{\mathcal{F}}{\longleftrightarrow}\|\boldsymbol{\omega}\|^{\gamma} \hat{f}(\boldsymbol{\omega})
$$

where $\mathcal{S}^{\prime}$ denotes Schwartz's space of tempered distributions. The usual discrete finite-difference counterpart of the $d$-dimensional Laplacian $\Delta$ is the digital filter $\Delta_{\mathrm{d}}$, whose frequency response is

$$
-\hat{\Delta}_{\mathrm{d}}(\boldsymbol{\omega})=4\|\sin (\boldsymbol{\omega} / 2)\|^{2} \triangleq 4 \sum_{l=1}^{d} \sin ^{2}\left(\frac{\omega_{l}}{2}\right) .
$$

One can also obtain a discrete counterpart of the fractional Laplacian by considering the $(\gamma / 2)$ th power of this expression.

We like to view the polyharmonic B-splines as the functions that link the continuous and discrete versions of these (fractional) operators, e.g.,

$$
\forall f \in \mathcal{S}^{\prime}, \quad\left(-\Delta_{\mathrm{d}}\right)^{\frac{\gamma}{2}} f=\beta_{\gamma} *(-\Delta)^{\frac{\gamma}{2}} f .
$$

Manuscript received June 26, 2008; revised August 14, 2008. This work was supported in part by the Swiss National Science Foundation under Grant 200020-109415 and in part by the Center for Biomedical Imaging of the Geneva-Lausanne Universities and the EPFL, as well as the foundations Leenaards and Louis-Jeantet. The associate editor coordinating the review of this manuscript and approving it for publication was Prof. Lisimachos Paul Kondi.

Y. Barbotin, D. Van De Ville, and M. Unser are with the Biomedical Imaging Group (BIG), Ecole Polytechnique Fédérale de Lausanne (EPFL), Lausanne, Switzerland.

T. Blu is with the Chinese University of Hong Kong, Shatin, Hong Kong.

Digital Object Identifier 10.1109/LSP.2008.2006714
This yields the Fourier-domain characterization of the polyharmonic B-spline of order $\gamma$, which will serve as our definition

$$
\hat{\beta}_{\gamma}(\boldsymbol{\omega})=\frac{\left(-\hat{\Delta}_{\mathrm{d}}\right)^{\frac{\gamma}{2}}(\boldsymbol{\omega})}{(-\hat{\Delta})^{\frac{\gamma}{2}}(\boldsymbol{\omega})}=\left(\frac{\|\sin (\boldsymbol{\omega} / 2)\|}{\|\boldsymbol{\omega} / 2\|}\right)^{\gamma} .
$$

Note that this formula involves the $\gamma$ th power of a sinc-like function which is bounded since the numerator tempers the singularity at $\boldsymbol{\omega}=\mathbf{0}$. Also, when $d=1$ and $\gamma$ is even, we recover the classical symmetric polynomial B-splines.

The polyharmonic B-splines were introduced by Rabut as a proper, nonseparable generalization of the univariate B-splines [2]. The key point is that they generate shift-invariant bases for the whole family of Duchon's thin-plate splines when the data points are taken on a uniform grid [3]. They also satisfy scaling relations that make them ideal candidates for the construction of various types of nonseparable multidimensional wavelet bases [4], [5]. Thus, by analogy with what has been achieved in 1-D [6], the polyharmonic B-splines can provide the primary building blocks for designing multidimensional digital filtering algorithms for a whole variety of spline-based signal processing tasks including interpolation, least-squares approximation, the optimal estimation of fractal-like processes [7], and nonseparable wavelet transforms [4], [8]. In principle, these operations can all be implemented in the Fourier domain provided that one has an efficient mechanism for evaluating the autocorrelation (or Gram) filter, which plays a central role in the B-spline formulation. The two equivalent forms of this filter are

$$
\begin{aligned}
A_{\gamma}\left(e^{j \boldsymbol{\omega}}\right) & =\sum_{\mathbf{n} \in \mathbb{Z}^{d}} e^{-j\langle\boldsymbol{\omega}, \mathbf{n}\rangle} \beta_{2 \gamma}(\mathbf{n}) \\
& =\sum_{\mathbf{k} \in \mathbb{Z}^{d}}\left|\hat{\beta}_{\gamma}(\boldsymbol{\omega}+2 \mathbf{k} \pi)\right|^{2} .
\end{aligned}
$$

Unfortunately, none of these formulas lends itself to an easy numerical determination, and this has been a major obstacle for the deployment of polyharmonic spline techniques so far. The main difficulty stems from the fact that the polyharmonic B-splines are not compactly supported and that they do not admit simple space-domain expressions, which essentially rules out the use of (2) - the preferred formula for the (nonfractional) univariate case. The only option left is then to use (3) in combination with (1). The practical problem here is that the summation is over a multidimensional lattice and that the convergence of the truncated series is slow, especially for lower values of $\gamma$.

The purpose of this letter is to propose an alternative to the truncated series approximation that is computationally much more favorable. The primary idea is to relate the infinite sum 
in (3) to the Epstein zeta function and to then take advantage of computational techniques that were developed in computational physics and crystallography in particular [9]. Our approach owes a lot to the work of Crandall who proposed an algorithm for the fast evaluation of general Epstein zeta functions; his method is available online but not published otherwise [10].

\section{Computational Methods}

\section{A. Truncated-Sum Method}

The straightforward approach to calculate the autocorrelation filter $A_{\gamma}\left(e^{j \boldsymbol{\omega}}\right)$ is by taking a finite number of terms in (3), e.g., those within the hypersphere $\|\mathbf{k}\| \leq R$. It can be readily proved that such a procedure has a residual error that is $\mathcal{O}\left(R^{-2 \gamma+d / 2}\right)$; as $\gamma \rightarrow((d / 2))^{+}$, the decay of the error with $R$ stalls and the sum in (3) eventually diverges.

In $1-\mathrm{D}$, the convergence of the sum can be improved by way of an accelerated partial-sum formula [11] as follows:

$$
\begin{aligned}
A_{\gamma}\left(e^{j \omega}\right) \approx & {\left[\sum_{k=-R}^{R}\left|\frac{2 \sin (\omega / 2)}{\omega+2 \pi k}\right|^{2 \gamma}\right]+\left|\frac{\sin (\omega / 2)}{\pi R}\right|^{2 \gamma} } \\
& \times\left[\frac{2 R}{2 \gamma-1}-1+\frac{\gamma\left(\frac{2}{3} \pi^{2}+\omega^{2}\right)}{2 \pi^{2} R}-\frac{\gamma(2 \gamma+1) \omega^{2}}{4 \pi^{2} R^{2}}\right]
\end{aligned}
$$

which holds for $|\omega| \leq \pi$ and improves the remainder to $\mathcal{O}\left(R^{-2 \gamma-3}\right)$, instead of $\mathcal{O}\left(R^{-2 \gamma+1}\right)$ without the correction term.

While this type of acceleration technique is quite powerful in $1-\mathrm{D}$, it is much harder to apply in higher dimensions. It is difficult to derive the correction terms, not to mention the fundamental limitation that the convergence remains polynomial and slows down as $d$ increases for a fixed $\gamma$.

\section{B. Sum of Incomplete Gamma (iG) Functions}

Inspired by Crandall's approach for the fast evaluation of Epstein zeta functions, we propose to express the infinite sum in (3) as a sum of incomplete Gamma functions. To that end, we first rewrite the autocorrelation filter as

$$
A_{\gamma}\left(e^{j \boldsymbol{\omega}}\right)=\left(-\hat{\Delta}_{\mathrm{d}}\right)^{\gamma}(\boldsymbol{\omega}) \sum_{\mathbf{k} \in \mathbb{Z}^{d}} \frac{1}{\|\boldsymbol{\omega}+2 \pi \mathbf{k}\|^{2 \gamma}}
$$

where we have taken advantage of the fact that the digital filter $\hat{\Delta}_{\mathrm{d}}^{\gamma}(\boldsymbol{\omega})$ corresponding to the $\gamma$ th Laplacian is $2 \pi$-periodic.

Proposition 1: The autocorrelation filter can be rephrased as an infinite sum of incomplete Gamma functions. Specifically, for $\boldsymbol{\omega} \in]-\pi, \pi]^{d} \backslash\{\mathbf{0}\}$, we have

$$
\begin{aligned}
A_{\gamma}\left(e^{j \boldsymbol{\omega}}\right)= & \left(-\hat{\Delta}_{\mathrm{d}}\right)^{\gamma}(\boldsymbol{\omega})(2 \pi)^{-2 \gamma} \Gamma(\gamma)^{-1} \\
& \times\left[\frac{\pi^{\gamma}}{n / 2-\gamma}+\left\|\frac{\boldsymbol{\omega}}{2 \pi}\right\|^{-2 \gamma} \Gamma\left(\gamma, \pi\left\|\frac{\boldsymbol{\omega}}{2 \pi}\right\|^{2}\right)\right. \\
& +\sum_{\mathbf{k} \in \mathbb{Z}^{d} \backslash\{\mathbf{0}\}}\left\|\frac{\boldsymbol{\omega}}{2 \pi}-\mathbf{k}\right\|^{-2 \gamma} \Gamma\left(\gamma, \pi\left\|\frac{\boldsymbol{\omega}}{2 \pi}-\mathbf{k}\right\|^{2}\right) \\
& \left.+\pi^{2 \gamma-\frac{d}{2}} \cos (\langle\mathbf{k}, \boldsymbol{\omega}\rangle)\|\mathbf{k}\|^{2 \gamma-d} \Gamma\left(\frac{d}{2}-\gamma, \pi\|\mathbf{k}\|^{2}\right)\right]
\end{aligned}
$$

where $\Gamma(\alpha)$ and $\Gamma(\alpha, x)$ are the regular and incomplete Gamma function, respectively. For $\boldsymbol{\omega}=\mathbf{0}$, one simply gets $A_{\gamma}(\mathbf{1})=1$.

The proof of this result is given in the Appendix. Note that apart from the factor $\left(-\hat{\Delta}_{\mathrm{d}}\right)^{\gamma}(\boldsymbol{\omega})$, it corresponds to a special case of Crandall's formula for the generalized Epstein zeta function (cf. [10, eq. (2.2)])

$$
\Xi(s ; \mathbf{A}, \mathbf{c}, \mathbf{d})=\sum_{\mathbf{k} \in \mathbb{Z}^{d}} \frac{e^{2 \pi i \mathbf{c} \cdot \mathbf{A k}}}{\|\mathbf{A} \mathbf{k}-\mathbf{d}\|^{s}}
$$

with the specific choice of parameters $s=2 \gamma, \mathbf{A}=2 \pi \mathbf{I}, \mathbf{c}=\mathbf{0}$, $\mathrm{d}=-\omega$.

At first sight, the reformulation does not bring much improvement; i.e., it still involves an infinite number terms and these even look more complicated than before. The main point, however, is that the iG sum converges much faster than the previous one. Another convenient aspect is that efficient software implementations of iG functions are available.

\section{CONVERGENCE OF THE SUM OF IG FUNCTIONS}

We now show that the rate of convergence of the iG sum representation of $A_{\gamma}\left(e^{j \boldsymbol{\omega}}\right)$ is extremely favorable and independent upon $\gamma$-this is the key for obtaining a fast algorithm.

Proposition 2: Approximating $A_{\gamma}\left(e^{j \omega}\right)$ by truncating the $\mathrm{iG}$ expansion with a summation radius $\|\mathbf{k}\| \leq R$ has a remainder that is $\mathcal{O}\left(R^{d-3} e^{-\pi R^{2}}\right)$, for $\left.\left.\omega \in\right]-\pi, \pi\right]^{d}$.

Proof: We first bound the decay of the incomplete Gamma function for large $x$ as follows:

$\Gamma(\alpha, x)=\int_{x}^{\infty} t^{\alpha-1} e^{-t} d t \approx \sum_{k=\lfloor x\rfloor}^{\infty} k^{\alpha-1} e^{-k}+\mathcal{O}\left(x^{\alpha-1} e^{-x}\right)$.

The first term is justifiable because the integrand is monotonically decaying and positive. The error-term behavior is derived by noticing that $(k+1)^{\alpha-1} e^{-(k+1)}<(1 / 2)\left(k^{\alpha-1} e^{-k}\right)$

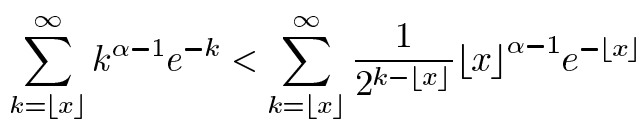

$$
\begin{aligned}
& <2 x^{\alpha-1} e^{-x} \\
& \sim \mathcal{O}\left(x^{\alpha-1} e^{-x}\right) \text {. }
\end{aligned}
$$

So we have $\Gamma(\alpha, x) \sim \mathcal{O}\left(x^{\alpha-1} e^{-x}\right)$. Both terms of the sum in Proposition 1 are of the form $\sum_{\mathbf{k} \in \mathbb{Z}^{d}}\left(\Gamma\left(\alpha, \pi\|\mathbf{k}\|^{2}\right) / \pi\|\mathbf{k}\|^{2 \alpha}\right)$, $\alpha \in\{\gamma, d / 2-\gamma\}$. The summation of the terms with index $\|\mathbf{k}\|>R$ therefore has a magnitude of

$$
\mathcal{O}\left(\int_{R}^{\infty} r^{d-3} e^{-\pi r^{2}} d r+\mathcal{O}\left(R^{d-3} e^{-\pi R^{2}}\right)\right)
$$

where we switched to spherical coordinates in the continuous domain.

The above integral is quite familiar; i.e., the substitution $t=$ $\pi r^{2}$ reveals the incomplete Gamma function, which decays as previously shown

$$
\begin{aligned}
\int_{R}^{\infty} r^{d-3} e^{-\pi r^{2}} d r & =\frac{\pi^{1-d / 2}}{2} \Gamma\left(\frac{d}{2}-1, \pi R^{2}\right) \\
& \sim \mathcal{O}\left(R^{d-4} e^{-\pi R^{2}}\right) .
\end{aligned}
$$


TABLE I

RadiUs $R$ NECESSARY to OBTAIN A GIVEN PRECISION

\begin{tabular}{cl|ccc|ccc}
\hline \hline & & \multicolumn{3}{|c|}{ truncated-sum } & \multicolumn{3}{c}{ iG } \\
& & $10^{-6}$ & $10^{-12}$ & $10^{-15}$ & $10^{-6}$ & $10^{-12}$ & $10^{-15}$ \\
\hline \multirow{4}{*}{$\gamma$} & 7 & $<5$ & $<5$ & 5 & 2 & 3 & 3.5 \\
& 4.5 & $<5$ & 10 & 100 & 1.5 & 2.9 & 3.5 \\
& 3 & 10 & 250 & $>500$ & 1.5 & 2.25 & 3.5 \\
& 2 & 250 & $>500$ & $>500$ & 1 & 2.25 & 2.9 \\
\hline \hline
\end{tabular}

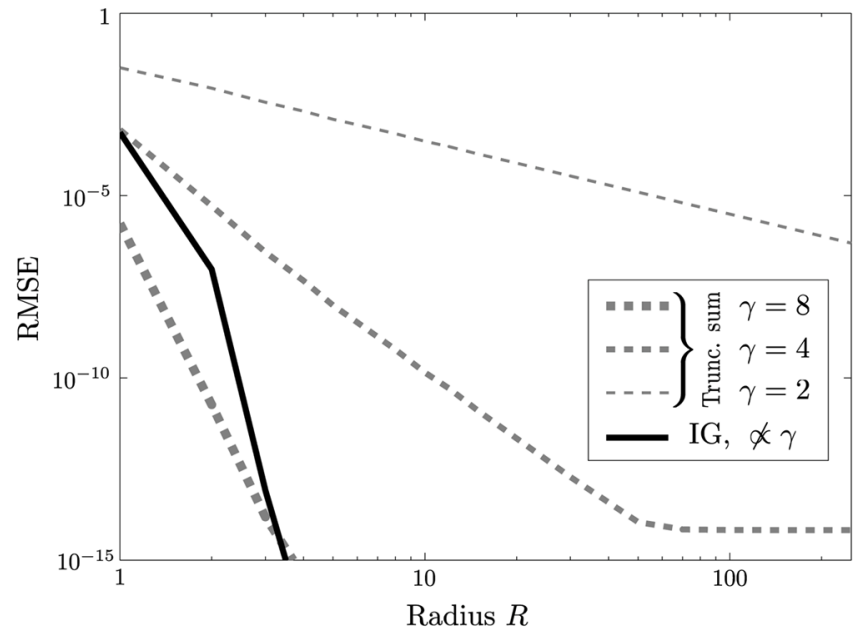

(a)

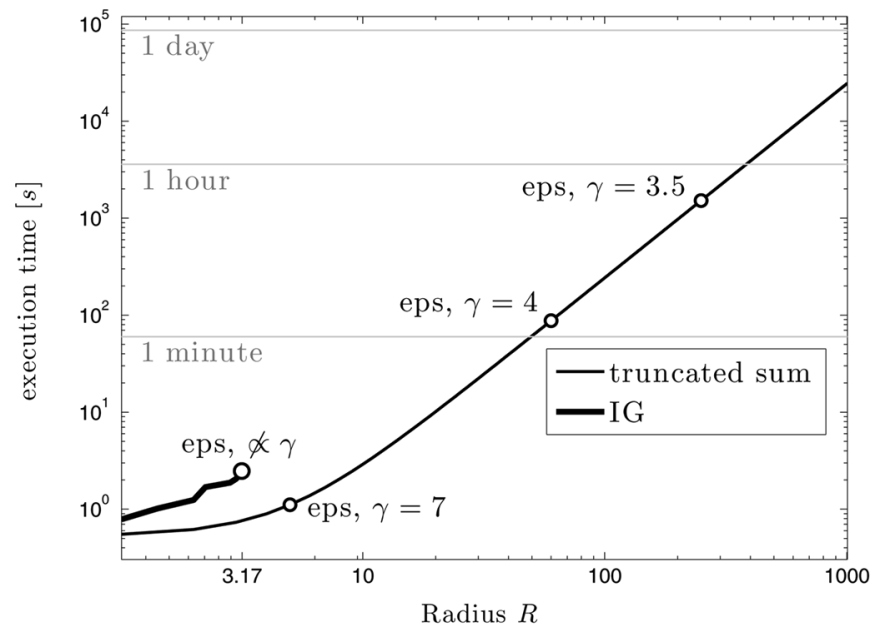

(b)

Fig. 1. (a) RMSE as a function of the radius $R$. The error for the IG method is independent of $\gamma$ and quickly drops below machine precision. (b) Execution time as a function of the radius $R$, including the levels when machine precision is reached.

In conclusion, we see that the truncated version of the sum in Proposition 1 with $\|\mathbf{k}\| \leq R$ leads to an approximation error that decays like $\mathcal{O}\left(R^{d-3} e^{-\pi R^{2}}\right)$.

We have thereby established that the convergence of the truncated iG sum is exponential with respect to the square-norm of the summation radius. This represents a significant gain with respect to the prior inverse-polynomial behavior. Furthermore, it no longer depends on the order $\gamma$ while the influence of the number of dimensions $d$ has also become negligible. This is an important point, as in practice the autocorrelation filter often needs to be evaluated for values of $\gamma$ that are relatively close to $d / 2$.

\section{Performance Evaluation}

We implemented in Matlab both the naive truncated-sum method and the iG-based one for the 2-D case. The autocorrelation filter was computed on a $512 \times 512$ uniform grid in $(-\pi, \pi]^{2}$. Both implementations take advantage of the symmetries, reducing the number of computed samples by a factor 8 (approx.). It is important to establish the ground-truth as a reference for the algorithm; this was obtained using the truncated-sum method for $\gamma \geq 4$ and the iG method otherwise. In each case, we added terms until the maximal contribution dropped below $10^{-15}$, which is the machine precision. ${ }^{1}$

In Fig. 1(a), we plot the root-mean-squared error (RMSE) between the ground truth and the obtained solution as a function of the order $\gamma$ and the summation radius $R$. The truncated-sum method shows a large dependence on the order, as expected. The method also becomes inaccurate for low $\gamma$. For the iG method, machine precision was reached already for $R=4$, even for $\gamma$ very close to 1 (see Table I). It is also (almost) independent of the order.

In Fig. 1(b), we show the execution time. Measured on a desktop PC (Core 2 duo $2.13 \mathrm{GHz}$ ), the iG method reaches machine precision in a steady $3 \mathrm{~s}$, independent from $\gamma$. The truncated-sum method does better for $\gamma>6$. However, for lower $\gamma$, it quickly becomes impracticable (e.g., $\gamma=4$ runs for roughly $45 \mathrm{~min}$ ).

\section{CONCLUSION}

The proposed algorithm provides us with a fast implementation of one of the key components of a multidimensional spline toolbox. Thanks to it, we can now transpose most of the digital filtering techniques available for the traditional polynomial splines to the nonseparable polyharmonic framework. We have already taken advantage of the method to obtain an efficient and accurate Fourier-domain implementation of a whole family of polyharmonic-spline wavelet transforms which will be made available soon.

\section{APPENDIX \\ PROOF OF THE IG SUM FORMULA}

While the result can be obtained from Crandall's formula for Epstein zeta functions [10], we provide a complete, self-contained proof that is aimed at a signal processing audience.

Proof: The Laplacian filter $\left(-\hat{\Delta}_{\mathrm{d}}\right)^{\gamma}(\boldsymbol{\omega})$ can be computed easily and only plays a role on the convergence of the series for $\boldsymbol{\omega}=\mathbf{0}$, in which case we get $A_{\gamma}(\mathbf{1})=1$. Therefore, we focus on the computation of $S_{\gamma}\left(e^{j \boldsymbol{\omega}}\right) \triangleq \sum_{\mathbf{k} \in \mathbb{Z}^{d}}\left(1 /\|\boldsymbol{\omega}+2 \mathbf{k} \pi\|^{2 \gamma}\right)$. We proceed in five steps and perform the substitution $s=2 \gamma$ and $\mathbf{f}=\boldsymbol{\omega} /(2 \pi)$ to simplify the notation.

Step 1 (Summation Terms as Euler Integrals): Euler's integral formula for the Gamma function is (cf. [12])

$$
\Gamma(z)=\eta^{z} \int_{0}^{\infty} t^{z-1} e^{-\eta t} d t, \quad \Re z, \Re \eta>0 .
$$

${ }^{1}$ Matlab's IEEE double precision arithmetics were used ( 8 bytes representation). 
By taking $\eta=\|\mathbf{f}-\mathbf{k}\|^{2}$ and $z=s / 2$, we obtain

$$
\frac{1}{\|\mathbf{f}-\mathbf{k}\|^{s}}=\frac{1}{\Gamma(s / 2)} \int_{0}^{\infty} t^{s / 2-1} e^{-t\|\mathbf{f}-\mathbf{k}\|^{2}} d t
$$

Step 2 (Splitting the Integral): Because the integrand is in $L_{1}$ and the autocorrelation sum is absolutely convergent, one may interchange the summation and the integration. The integral is then split in two parts as follows:

$$
\begin{aligned}
(2 \pi)^{s} & \Gamma(s / 2) S_{\gamma}(2 \pi \mathbf{f}) \\
= & \int_{0}^{\infty} t^{s / 2-1}\left[\sum_{\mathbf{k} \in \mathbb{Z}^{d}} e^{-t\|\mathbf{f}-\mathbf{k}\|^{2}}\right] d t \\
= & \int_{0}^{\pi} t^{s / 2-1} \sum_{\mathbf{k} \in \mathbb{Z}^{d}} e^{-t\|\mathbf{f}-\mathbf{k}\|^{2}} d t \\
& +\int_{\pi}^{\infty} t^{s / 2-1} \sum_{\mathbf{k} \in \mathbb{Z}^{d}} e^{-t\|\mathbf{f}-\mathbf{k}\|^{2}} d t .
\end{aligned}
$$

Step 3 (The $[\pi \infty]$ Integral): The right term in (6) immediately yields a sum of incomplete Gamma functions as follows:

$$
\begin{aligned}
\int_{\pi}^{\infty} t^{s / 2-1} \sum_{\mathbf{k} \in \mathbb{Z}^{d}} e^{-t\|\mathbf{f}-\mathbf{k}\|^{2}} d t \\
=\sum_{\mathbf{k} \in \mathbb{Z}^{d}}\|\mathbf{f}-\mathbf{k}\|^{-s} \int_{\pi\|\mathbf{f}-\mathbf{k}\|^{2}}^{\infty} r^{s / 2-1} e^{-r} d r \\
=\sum_{\mathbf{k} \in \mathbb{Z}^{d}}\|\mathbf{f}-\mathbf{k}\|^{-s} \Gamma\left(s / 2, \pi\|\mathbf{f}-\mathbf{k}\|^{2}\right) .
\end{aligned}
$$

Step 4 (The $[0 \pi]$ Integral): More effort is required to put the left term of (6) in a computable form. We perform the change of variables $r=1 / t$ as follows:

$$
\begin{aligned}
& \int_{0}^{\pi} t^{s / 2-1}\left[\sum_{\mathbf{k} \in \mathbb{Z}^{d}} e^{-t\|\mathbf{f}-\mathbf{k}\|^{2}}\right] d t \\
&=\int_{\frac{1}{\pi}}^{\infty} r^{-s / 2-1}\left[\sum_{\mathbf{k} \in \mathbb{Z}^{d}} e^{-\frac{1}{r}\|\mathbf{f}-\mathbf{k}\|^{2}}\right] d r .
\end{aligned}
$$

Step 5 (Poisson Summation Formula): Applying Poisson's summation formula, we get

$$
\sum_{\mathbf{k} \in \mathbb{Z}^{d}} e^{-t \mid \mathbf{f}-\mathbf{k} \|^{2}}=(\pi r)^{\frac{d}{2}} \sum_{\mathbf{n} \in \mathbb{Z}^{d}} e^{-j 2 \pi\langle\mathbf{n}, \mathbf{f}\rangle} e^{-\pi^{2} r\|\mathbf{n}\|^{2}}
$$

which allows us to rewrite the $[0 \pi]$ integral as (recall $s>d$ )

$$
\begin{aligned}
\int_{\frac{1}{\pi}}^{\infty} r^{-s / 2-1}\left[\sum_{\mathbf{k} \in \mathbb{Z}^{d}} e^{-\frac{1}{r}\|\mathbf{f}-\mathbf{n}\|^{2}}\right] d r \\
=\pi^{d / 2} \sum_{\mathbf{n} \in \mathbb{Z}^{d} \backslash\{\mathbf{0}\}} e^{-j 2 \pi\langle\mathbf{n}, \mathbf{f}\rangle} \int_{\frac{1}{\pi}}^{\infty} r^{\frac{d-s}{2}-1} e^{-r \pi^{2}\|\mathbf{n}\|^{2}} d r \\
+\pi^{d / 2} \int_{\frac{1}{\pi}}^{\infty} r^{\frac{d-s}{2}-1} d r \\
=\pi^{s-d / 2} \sum_{\mathbf{n} \in \mathbb{Z}^{d} \backslash\{\mathbf{0}\}} \frac{e^{-j 2 \pi\langle\mathbf{n}, \mathbf{f}\rangle}}{\|\mathbf{n}\|^{d-s}} \int_{\pi\|\mathbf{n}\|^{2}}^{\infty} t^{\frac{d-s}{2}-1} e^{-t} d t \\
+\frac{2 \pi^{s / 2}}{d-s} \sum_{\mathbf{n} \in \mathbb{Z}^{d} \backslash\{\mathbf{0}\}} \frac{e^{-j 2 \pi\langle\mathbf{n}, \mathbf{f}\rangle}}{\|\mathbf{n}\| \|^{d-s}} \Gamma\left(\frac{d-s}{2}, \pi\|\mathbf{n}\|^{2}\right)+\frac{2 \pi^{s / 2}}{d-s} .
\end{aligned}
$$

As expected, the result is real-valued since the terms with indices $\mathbf{n}$ and $-\mathbf{n}$ are complex conjugates of each other.

Putting the pieces together yields the iG sum formula.

\section{REFERENCES}

[1] I. M. Gel'fand and G. E. Shilov, "Properties and operations," in Generalized Functions. New York: Academic, 1964, vol. I, translated by E. Saletan.

[2] C. Rabut, "Elementary $m$-harmonic cardinal B-splines," Numer. Alg., vol. 2, pp. 39-62, 1992.

[3] J. Duchon, "Splines minimizing rotation-invariant semi-norms in Sobolev spaces," in Constructive Theory of Functions of Several Variables, W. Schempp and K. Zeller, Eds. Berlin, Germany: Springer-Verlag, 1977, pp. 85-100.

[4] D. Van De Ville, T. Blu, and M. Unser, "Isotropic polyharmonic B-splines: Scaling functions and wavelets," IEEE Trans. Image Process., vol. 14, no. 11, pp. 1798-1813, Nov. 2005.

[5] B. Bacchelli, M. Bozzini, C. Rabut, and M.-L. Varas, "Decomposition and reconstruction of multidimensional signals using polyharmonic pre-wavelets," Appl. Comput. Harmon. Anal., vol. 18, no. 3, pp. 282-299, May 2005.

[6] M. Unser, "Splines: A perfect fit for signal and image processing," IEEE Signal Process. Mag., vol. 16, no. 6, pp. 22-38, Nov. 1999.

[7] S. Tirosh, D. Van De Ville, and M. Unser, "Polyharmonic smoothing splines and the multidimensional Wiener filtering of fractal-like signals," IEEE Trans. Image Process., vol. 15, no. 9, pp. 2616-2630, Sep. 2006.

[8] B. Forster, T. Blu, D. Van De Ville, and M. Unser, "Shift-invariant spaces from rotation-covariant functions," Appl. Comput. Harmon. Anal., vol. 25, no. 2, pp. 240-265, Sep. 2008.

[9] S. Tyagi, "New series representation for madelung constant," Prog. Theoret. Phys., vol. 114, pp. 517-521, 2005.

[10] R. C. Crandall, Fast Evaluation of Epstein Zeta Functions, 1998. [Online]. Available: http://people.reed.edu/crandall/papers/epstein.pdf.

[11] M. Unser and T. Blu, "Self-similarity: Part I-Splines and operators," IEEE Trans. Signal Process., vol. 55, no. 4, pp. 1352-1363, Apr. 2007.

[12] M. Abramowitz and I. A. Stegun, Handbook of Mathematical Functions With Formulas, Graphs, and Mathematical Tables, 9th ed. New York: Dover, 1964 